\title{
Smeared versus localised sources in flux compactifications
}

\author{
Johan Blåbäck, ${ }^{a}$ Ulf H. Danielsson, ${ }^{a}$ Daniel Junghans, ${ }^{b}$ Thomas Van Riet, ${ }^{a}$ \\ Timm Wrase ${ }^{b, c}$ and Marco Zagermann ${ }^{b, d}$ \\ ${ }^{a}$ Institutionen för fysik och astronomi, Uppsala Universitet, \\ Box 803, SE-751 08 Uppsala, Sweden \\ ${ }^{b}$ Institut für Theoretische Physik \&, Center for Quantum Engineering and Spacetime Research, \\ Leibniz Universität Hannover, \\ Appelstraße 2, 30167, Hannover, Germany \\ ${ }^{c}$ Department of Physics, Cornell University, \\ Ithaca, NY 14853, U.S.A. \\ ${ }^{d}$ Kavli Institute for Theoretical Physics, \\ Santa Barbara, CA 93106, U.S.A. \\ E-mail: johan.blaback@fysast.uu.se, ulf.danielsson@fysast.uu.se, \\ daniel.junghans@itp.uni-hannover.de, thomas.vanriet@fysast.uu.se, \\ timm.wrase@cornell.edu, marco.zagermann@itp.uni-hannover.de
}

ABSTRACT: We investigate whether vacuum solutions in flux compactifications that are obtained with smeared sources (orientifolds or D-branes) still survive when the sources are localised. This seems to rely on whether the solutions are BPS or not. First we consider two sets of BPS solutions that both relate to the GKP solution through T-dualities: $(p+1)$-dimensional solutions from spacetime-filling $\mathrm{O} p$-planes with a conformally Ricciflat internal space, and $p$-dimensional solutions with $\mathrm{O} p$-planes that wrap a 1-cycle inside an everywhere negatively curved twisted torus. The relation between the solution with smeared orientifolds and the localised version is worked out in detail. We then demonstrate that a class of non-BPS $\mathrm{AdS}_{4}$ solutions that exist for IASD fluxes and with smeared D3branes (or analogously for ISD fluxes with anti-D3-branes) does not survive the localisation of the (anti) D3-branes. This casts doubts on the stringy consistency of non-BPS solutions that are obtained in the limit of smeared sources.

KEYwORDs: Flux compactifications, Superstring Vacua, D-branes

ARXIV EPRINT: 1009.1877 


\section{Contents}

1 Introduction 1

2 Type II supergravity 3

3 BPS solutions with Ricci-flat internal space 4

3.1 The smeared solutions 4

$\begin{array}{lll}3.2 & \text { The localised solutions } & 6\end{array}$

$\begin{array}{lll}4 & \text { BPS solutions with negatively curved twisted tori } & 8\end{array}$

$\begin{array}{lr}\text { 4.1 The smeared solutions } & 9\end{array}$

$\begin{array}{ll}4.2 & \text { A simple example } \\ & 11\end{array}$

$\begin{array}{lll}4.3 & \text { The localised solutions } & 12\end{array}$

5 Non-BPS solutions $\quad \mathbf{1 5}$

$\begin{array}{lll}5.1 & \text { The smeared solutions } & 15\end{array}$

$\begin{array}{ll}5.2 \text { A simple example } & 16\end{array}$

$\begin{array}{ll}\text { 5.3 The failure to localise? } & 16\end{array}$

$\begin{array}{llr}6 & \text { Discussion } & 18\end{array}$

\section{Introduction}

Many compactifications that give rise to vacua with phenomenologically appealing properties feature spacetime-filling sources such as orientifold planes or D-branes. In most cases these solutions are derived in the limit that the sources are smeared, although some localised solutions are known, see e.g. [1-3]. Smearing means that delta function sources in the equations of motion are replaced with specific regular functions that integrate to the same value.

Apart from simplifying the task of finding solutions, reductions with smeared sources may allow for consistent truncations in certain compactifications on group spaces or coset manifolds (see e.g. [4, 5]). However, an orientifold plane is defined through its involution and a D-brane by its boundary conditions, which makes them really localised objects. It is therefore important to study the corrections to a smeared solution that arise upon localising an orientifold plane or a D-brane. A notable difference between smeared and localised solutions is that the equations of motion, in the localised case, necessarily imply that the spacetime is warped (which can be appealing to solve the hierarchy problem [6]). 
One way to incorporate the changes that arise upon localising a source is through "warped effective field theory" [7-18], in which one derives the correction to the fourdimensional effective action. Another way, which we are pursuing in this paper, is to work directly with the ten-dimensional equations of motion.

There are only few known solutions with localised sources, and we therefore first generalise some existing solutions to other spacetime dimensions. All the solutions turn out to be T-dual to the known four-dimensional solution of [1]. In the smeared limit these solutions either have an internal space that is Ricci-flat, being the $D$-dimensional generalisation of the GKP solution [1], or the solutions have a negatively curved twisted torus as internal space, being the $D$-dimensional generalisation of some solutions given in [19] (which themselves are T-dual to the GKP solution, see also [20]). The solutions on the twisted tori of [19] were obtained in the smeared limit and a discussion on their localisation was given in $[2,3]$. Here we discuss the $D$-dimensional generalisation of these solutions very explicitly from the point of view of the 10-dimensional equations of motion. Furthermore we do not assume flux configurations that are necessarily supersymmetric, in contrast to most references on solutions from twisted tori. However, the solutions will be BPS, in the same sense that the GKP solution is BPS but not necessarily supersymmetric. The phenomenon of BPS but non-susy solutions has been given a higher-dimensional interpretation in [21], where the BPSness turns out to be directly related to the existence of brane calibrations even in absence of SUSY.

Apart from providing a larger playground for studying localisation effects there is a general interest in constructing the $D$-dimensional generalisation of the four-dimensional string landscape. Partly because some stringy consistency issues might be easier to deal with in lower dimensions (see e.g. the recent discussion in [22]) and partly because the landscape is really featuring vacua of all kinds of dimensions and there might be transitions between vacua of various dimensions (see e.g. [23]).

Our motivation for this paper comes from the interesting observation made in [24] that string/M theory compactifications, at tree-level, using manifolds whose curvature is everywhere negative, must have significant warping. Since part of our solutions, in the smeared limit, have negatively curved spaces we can address this issue in concrete examples. The reason that significant warping is required is that, for unwarped metrics, one can show that negative curvature in the internal space requires a source of energy momentum with negative tension at every point. Hence, imagine that in the localised case there exists a regime in which warping can be neglected compared to the fluxes. Then in that regime we require a source of negative tension at every point in the internal space. This leads to a contradiction since this regime is by definition far away from the delta-like orientifold source, where warping is strong. We elaborate further on this argument and illustrate it with our simple examples. Specifically, we want to emphasize the rôle of the BPS condition in achieving localisation. Furthermore the T-duality chain of the BPS solutions shows that the argument of [24] for large warping corrections to solutions with negatively curved internal spaces extends also to solutions with Ricci-flat internal spaces and $H$-flux. As an important application of our results, we show that the properly integrated negative curvature of the smeared twisted tori solutions stays negative upon the localization of the O-planes, contrary to some naive expectations. 
The main conclusion of our examples is that the individual localisation corrections ${ }^{1}$ cancel against each other in the effective potential for certain BPS solutions (along the lines of [7]). However, there is in general no reason to expect an analogous cancelation of localisation effects for non-BPS solutions. As an illustration, we explicitly construct, in section 5, AdS solutions with smeared sources that are non-BPS by going beyond the ISD flux configurations. Then we show how $\mathrm{AdS}_{4}$ solutions with D3-branes and imaginary anti-self-dual (IASD) fluxes (or analogously anti-D3-branes with ISD fluxes) do not survive localisation. We discuss the possible implications of our results in section 6 .

\section{Type II supergravity}

To establish our notation and conventions, we present the equations of motion for type IIA/B supergravity with $\mathrm{O} p$-sources in Einstein frame ( $\mathrm{D} p$-branes will be considered in section 5). We use the conventions of [25] but go to Einstein frame and change the sign of $H$ (see also appendix A of [26]). Compared to [25] our solutions with $\mu_{p}>0$ correspond to O-planes for $p=2,3,6$ and anti-O-planes for $p=1,4,5$ and analogously for D-branes which have $\mu_{p}<0$. Note that one can always flip the sign of all RR-fields, which leaves the closed string action invariant and maps O-planes/D-branes to anti-O-planes/anti-Dbranes. Throughout the paper $a, b$ are 10D indices; $\mu, \nu$ are along the orientifold plane and $i, j$ are transverse to it. The common bosonic sector contains the metric $g_{a b}$, the dilaton $\phi$ and the $H$ field strength. The RR sector of (massive) type IIA consist of the $\left(F_{0},\right) F_{2}, F_{4}$ field strengths, whereas in IIB one has the field strengths $F_{1}, F_{3}, F_{5}$, with $F_{5}$ satisfying $F_{5}=\star F_{5}$.

The trace reversed Einstein equation is

$$
\begin{aligned}
R_{a b}= & \frac{1}{2} \partial_{a} \phi \partial_{b} \phi+\mathrm{e}^{-\phi}\left(\frac{1}{2}|H|_{a b}^{2}-\frac{1}{8} g_{a b}|H|^{2}\right) \\
& +\sum_{n \leq 5} \mathrm{e}^{\frac{5-n}{2} \phi}\left(\frac{1}{2\left(1+\delta_{n 5}\right)}\left|F_{n}\right|_{a b}^{2}-\frac{n-1}{16\left(1+\delta_{n 5}\right)} g_{a b}\left|F_{n}\right|^{2}\right)+\frac{1}{2}\left(T_{a b}^{l o c}-\frac{1}{8} g_{a b} T^{l o c}\right)
\end{aligned}
$$

where $\delta_{n 5}$ is the Kronecker delta, and $|A|_{a b}^{2} \equiv \frac{1}{(p-1) !} A_{a a_{2} \ldots a_{p}} A_{b}^{a_{2} \ldots a_{p}}, \quad|A|^{2} \equiv$ $\frac{1}{p !} A_{a_{1} \ldots a_{p}} A^{a_{1} \ldots a_{p}}$.

The non-vanishing part of the local stress tensor is given by ${ }^{2}$

$$
T_{\mu \nu}^{l o c}=\mathrm{e}^{\frac{p-3}{4} \phi} \mu_{p} g_{\mu \nu} \delta(O p), \quad \mu, \nu=0,1, \ldots, p,
$$

where $\mu_{p}$ is a positive number for an orientifold source and $\delta(O p)$ is a delta distribution with support on the $\mathrm{O} p$-plane world volume. The dilaton equation of motion is given by

$$
\nabla^{2} \phi=-\mathrm{e}^{-\phi} \frac{1}{2}|H|^{2}+\sum_{n \leq 5} \mathrm{e}^{\frac{5-n}{2} \phi} \frac{5-n}{4}\left|F_{n}\right|^{2}-\frac{p-3}{4} \mathrm{e}^{\frac{p-3}{4} \phi} \mu_{p} \delta(O p) .
$$

\footnotetext{
${ }^{1}$ Localisation corrections include, apart from the warp factor, corrections due to the dilaton that varies in the internal space and the non-zero field strength that is sourced by the orientifold or D-brane.

${ }^{2}$ Here and in the following, $\delta(O p)$ is meant to implicity also include sums of parallel $\mathrm{O} p$-planes.
} 
The Bianchi identities for the field strength are

$$
\begin{aligned}
\mathrm{d} H & =0, \\
\mathrm{~d} F_{n} & =H \wedge F_{n-2}-\mu_{8-n} \delta_{n+1}(O(8-n)),
\end{aligned}
$$

where $\delta_{n+1}(O(8-n))$ is shorthand for the normalized $(n+1)$ volume form transverse to the $\mathrm{O}(8-n)$ orientifold plane multiplied by $\delta(O(8-n))$. The equations of motion for the RR field strengths,

$$
\mathrm{d}\left(\mathrm{e}^{\frac{5-n}{2} \phi} \star F_{n}\right)=-\mathrm{e}^{\frac{3-n}{2} \phi} H \wedge \star F_{n+2}-(-1)^{\frac{n(n-1)}{2}} \mu_{n-2} \delta_{11-n}(O(n-2)),
$$

can be obtained from the RR Bianchi identities for $n>5$ upon employing the rule $\mathrm{e}^{(5-n) \phi / 2} F_{n}=(-1)^{(n-1)(n-2) / 2} \star F_{10-n}$.

Finally, the equation of motion for the $H$ field strength is given by

$$
\mathrm{d}\left(\mathrm{e}^{-\phi} \star H\right)=-\frac{1}{2} \sum_{n} \mathrm{e}^{\frac{5-n}{2} \phi} \star F_{n} \wedge F_{n-2}
$$

where the sum over $n$ includes all even/odd numbers up to 10 for IIA/IIB.

\section{BPS solutions with Ricci-flat internal space}

In this section, we consider a flux compactification down to $p+1$ dimensions with a spacetime-filling orientifold plane that has a pointlike extension in the internal space (i.e. an $\mathrm{O} p$-plane). For $p=3$ this is the famous GKP solution [1]. The generalisations we find here are written down for general $p=1, \ldots, 6$.

\subsection{The smeared solutions}

When we look for solutions with smeared orientifold sources, we assume that the dilaton, $\phi$, is constant and that the metric has the form of a direct product

$$
\phi=\phi_{0}, \quad \mathrm{~d} s_{10}^{2}=\mathrm{d} s_{p+1}^{2}+\mathrm{d} s_{9-p}^{2} .
$$

The non-zero form fields are $H$ and $F_{6-p}$. The rest of the RR-fields are identically zero.

The orientifold source enters the Einstein equation, the dilaton equation (unless $p=3$ ) and the Bianchi identity for the $F_{8-p}$ field. Smearing implies that the delta form function in the Bianchi identity is set equal to the normalized internal volume form, $\epsilon_{9-p}$, and in the Einstein and dilaton equations the delta functions are set equal to one. For a flat external space (Minkowski vacuum), this gives the following, external Einstein equation (in form notation)

$$
0=-\frac{1}{8} \mathrm{e}^{-\phi_{0}} \star_{9-p} H \wedge H-\frac{5-p}{16} \mathrm{e}^{\frac{p-1}{2} \phi_{0}} \star_{9-p} F_{6-p} \wedge F_{6-p}+\frac{7-p}{16} \mu_{p} \mathrm{e}^{\frac{p-3}{4} \phi_{0}} \epsilon_{9-p} .
$$

Since $F_{8-p}=0$, its Bianchi identity becomes

$$
0=H \wedge F_{6-p}-\mu_{p} \epsilon_{9-p}
$$


Combining (3.2) and (3.3) we can eliminate the source term to get

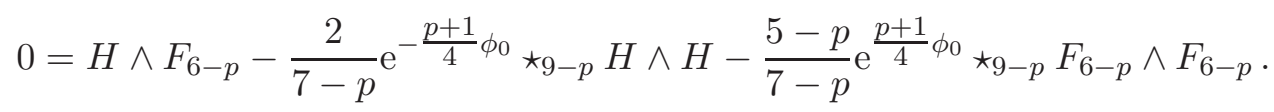

To solve the above relation we apply the following Ansatz

$$
F_{6-p}=(-1)^{p} \mathrm{e}^{-\frac{p+1}{4} \phi_{0}} \kappa \star_{9-p} H
$$

which provides a second order equation in $\kappa$. To later be able to solve the dilaton equation, it turns out that one solution has to be discarded. The remaining solution is

$$
F_{6-p}=(-1)^{p} \mathrm{e}^{-\frac{p+1}{4} \phi_{0}} \star_{9-p} H .
$$

For the special case of $p=3$ this is the so-called ISD condition on the $G$-flux [1]. For $p=1$ there is a subtlety because of the self-duality of $F_{5}$. The same derivation still applies as long as one carries the self-duality around, and the result is that (3.6) needs to be adjusted by adding the (10-dimensional) Hodge dual piece to the right hand side.

We will refer to the duality (3.6) as the BPS condition for reasons that become clear below. Note, that just as in GKP, the BPS condition does not necessarily imply supersymmetry. For constant dilaton the BPS condition equates the $H$ equation of motion to the $F_{6-p}$ Bianchi identity, and vice versa. Furthermore, using the BPS condition in (3.3) allows us to express the values of the fluxes in terms of $\mu_{p}$,

$$
\mu_{p}=\mathrm{e}^{-\frac{p+1}{4} \phi_{0}}|H|^{2}=\mathrm{e}^{\frac{p+1}{4} \phi_{0}}\left|F_{6-p}\right|^{2} .
$$

The dilaton equation of motion gives

$$
0=\nabla^{2} \phi_{0}=-\frac{1}{2} \mathrm{e}^{-\phi_{0}}|H|^{2}+\frac{p-1}{4} \mathrm{e}^{\frac{p-1}{2} \phi_{0}}\left|F_{6-p}\right|^{2}-\frac{p-3}{4} \mathrm{e}^{\frac{p-3}{4} \phi_{0}} \mu_{p} .
$$

The BPS relation (3.6) combines the $\left|F_{6-p}\right|^{2}$ term with the $|H|^{2}$ term, such that

$$
0=\frac{p-3}{4} \mathrm{e}^{-\phi_{0}}|H|^{2}-\frac{p-3}{4} \mathrm{e}^{\frac{p-3}{4} \phi_{0}} \mu_{p} .
$$

This is solved trivially for $p=3$ and reduces to $(3.7)$ for $p \neq 3$.

The internal Einstein equation is

$$
\begin{aligned}
R_{i j}= & \left(-\frac{1}{8} \mathrm{e}^{-\phi_{0}}|H|^{2}-\frac{5-p}{16} \mathrm{e}^{\frac{p-1}{2} \phi_{0}}\left|F_{6-p}\right|^{2}-\frac{p+1}{16} \mathrm{e}^{\frac{p-3}{4} \phi_{0}} \mu_{p}\right) g_{i j} \\
& +\frac{1}{2} \mathrm{e}^{-\phi_{0}}|H|_{i j}^{2}+\frac{1}{2} \mathrm{e}^{\frac{p-1}{2} \phi_{0}}\left|F_{6-p}\right|_{i j}^{2},
\end{aligned}
$$

which by the use of equations (3.6), (3.7) tells us that the internal space is Ricci-flat ${ }^{3}$

$$
R_{i j}=0 .
$$

\footnotetext{
${ }^{3}$ We have used the BPS condition (3.6) to rewrite $\left|F_{6-p}\right|_{i j}^{2}=\mathrm{e}^{-\frac{p+1}{2} \phi_{0}}\left(|H|^{2} g_{i j}-|H|_{i j}^{2}\right)$. This relation is not present in the case of $p=6$, since $F_{0}$ has no indices.
} 
Summary of the solution. The non-zero fields in the Ansatz for the smeared orientifold solution are (where $p=1, \ldots, 6)$

$$
\phi=\phi_{0}, \quad H, \quad F_{6-p}, \quad R_{i j} .
$$

This leads to a $(p+1)$-dimensional Minkowski solution provided the following conditions are satisfied

$$
\begin{aligned}
F_{6-p} & =(-1)^{p} \mathrm{e}^{-\frac{p+1}{4} \phi_{0}} \star_{9-p} H, \\
\mathrm{~d} H & =0, \quad \mathrm{~d} F_{6-p}=0, \\
\mu_{p} & =\mathrm{e}^{-\frac{p+1}{4} \phi_{0}}|H|^{2}=\mathrm{e}^{\frac{p+1}{4} \phi_{0}}\left|F_{6-p}\right|^{2}, \\
R_{i j} & =0 .
\end{aligned}
$$

For $p=1$ the Hodge dual piece needs to be added to the expression for $F_{5}$.

\subsection{The localised solutions}

We expect a localised orientifold to (i) induce a warping, (ii) source the $F_{8-p}$ field strength and (iii) lead to a dilaton that varies in the internal space. This can be seen from the standard $\mathrm{D} p$-brane solutions in asymptotically flat space. Therefore, if we consider a localised orientifold in a flux background, the solution should allow two limits; a limit in which the smeared flux background is found and a limit in which the $\mathrm{D} p$-brane solution in asymptotically flat space is found after eliminating the background fluxes. In that sense one could view the localised solutions, if one exists, as a superposition of two solutions. So, as argued, the non-zero fields are

$$
F_{8-p}, F_{6-p}, H, \phi .
$$

The metric Ansatz is given by

$$
\mathrm{d} s_{10}^{2}=\mathrm{e}^{2 a A} \mathrm{~d} \tilde{s}_{p+1}^{2}+\mathrm{e}^{2 b A} \mathrm{~d} \tilde{s}_{9-p}^{2},
$$

where $a$ and $b$ are some numbers to be determined later, and $A$ is a function of the internal coordinates $x^{i}$ and is called the warp factor. The external and internal metric are written as

$$
\mathrm{d} \tilde{s}_{p+1}^{2}=\tilde{g}_{\mu \nu} \mathrm{d} x^{\mu} \mathrm{d} x^{\nu}, \quad \mathrm{d} \tilde{s}_{9-p}^{2}=\tilde{g}_{i j} \mathrm{~d} x^{i} \mathrm{~d} x^{j} .
$$

So tildes are used when the warp factor dependence is taken out. This will also apply to covariant derivatives $(\tilde{\nabla})$, squares of tensors $\left(\tilde{T}^{2}\right)$ and so on.

There is an ambiguity in what we call the internal metric $\tilde{g}_{i j}$ since we can always absorb powers of $A$. Hence the number $b$ can be seen as a gauge choice. Nonetheless, when one considers how warping arises upon localising a source then the internal metric has an absolute meaning as the internal metric before localisation.

The Ricci tensor for the metric Ansatz (3.18) reads

$$
\begin{aligned}
R_{\mu \nu}= & \tilde{R}_{\mu \nu}-\mathrm{e}^{2(a-b) A} \tilde{g}_{\mu \nu}\left(a[(p+1) a+(7-p) b](\tilde{\partial} A)^{2}+a \tilde{\nabla}^{2} A\right), \\
R_{i j}= & \tilde{R}_{i j}-\left(b[(p+1) a+(7-p) b](\tilde{\partial} A)^{2}+b \tilde{\nabla}^{2} A\right) \tilde{g}_{i j} \\
& +[b((p+1) a+(7-p) b)-(p+1) a(a-b)] \partial_{i} A \partial_{j} A-[(p+1) a+(7-p) b] \tilde{\nabla}_{i} \partial_{j} A .
\end{aligned}
$$


There are no mixed components. The solution for the sources in flat space have

$$
(p+1) a+(7-p) b=0,
$$

which is what we assume from now on. We furthermore choose the normalisation of $A$ such that $a=1$. Note that this makes the expression for the Ricci tensor much simpler.

The Ansatz for the $F_{8-p}$ field strength is

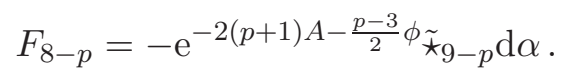

For $p=3$, we have to add the Hodge dual, and this coincides exactly with the Ansatz of GKP [1]. For $p=2$ there is also a subtlety since $F_{8-p}$ is dual to $F_{6-p}$. Therefore, for $p=2$ the dual of the above expression for $F_{6}$ needs to be added to the expression for $F_{4}$ we will now construct (see (3.28)).

We obtain two equations by combining the Bianchi identity for $F_{8-p}$ with the traced external Einstein equation to remove the source, and by combining the dilaton equation with the traced external Einstein equation to remove the source. After some algebra, these two equations can be combined into

$$
\begin{aligned}
\tilde{\nabla}^{2}\left(\mathrm{e}^{(p+1) A+\frac{p-3}{4} \phi}+(-1)^{p} \alpha\right)= & \mathrm{e}^{\frac{(p-3)^{2}}{p-7} A} \mathrm{e}^{\frac{p-3}{4} \phi} \tilde{R}_{p+1} \\
& +\mathrm{e}^{\frac{(p+1)(9-p)}{p-7} A-\frac{p-3}{4} \phi}\left|\partial\left(\mathrm{e}^{(p+1) A+\frac{p-3}{4} \phi}+(-1)^{p} \alpha\right)\right|^{2} \\
& +\frac{1}{2} \mathrm{e}^{\frac{(p+1)(p-5) A}{p-7}+\frac{3 p-5}{4} \phi}\left|F_{6-p}-(-1)^{p} \mathrm{e}^{-\frac{p+1}{4} \phi} \star_{9-p} H\right|^{2},
\end{aligned}
$$

where the squares in the last two term are with respect to the warped metric. Since the left hand side integrates to zero on a compact space, we find that $\tilde{R}_{p+1} \leq 0$. For Minkowski solutions $\left(\tilde{R}_{p+1}=0\right)$, both squares need to vanish, and we recover the duality condition (3.6) together with

$$
\alpha=(-1)^{p+1} \mathrm{e}^{(p+1) A+\frac{p-3}{4} \phi}+c s t .
$$

Note that these results are obtained without the use of any other equations than the traced external Einstein equation, the dilaton equation and the Bianchi identity for $F_{8-p}$. Let us therefore discuss how all other equations are solved. First of all we assume the Bianchi identities $\mathrm{d} H=0$ and $\mathrm{d} F_{6-p}=0$. A rather lengthy calculation. ${ }^{4}$ then shows that all the other equations are satisfied if the following conditions are met

$$
\begin{aligned}
\tilde{R}_{i j} & =0, \\
\tilde{\nabla}^{2}\left(\frac{4(p-3)}{7-p} A-\phi\right) & =0 \quad \Longrightarrow \quad \phi=\frac{4(p-3)}{7-p} A+\phi_{0}, \\
\tilde{\nabla}^{2} \mathrm{e}^{\frac{16}{p-7} A} & =-\mathrm{e}^{-\phi_{0}}|\tilde{H}|^{2}+\mathrm{e}^{\frac{p-3}{4} \phi_{0}} \mu_{p} \tilde{\delta}(O p) .
\end{aligned}
$$

\footnotetext{
${ }^{4}$ This computation goes along the same lines of the smeared case but is more involved. Useful identities are the expression for the Ricci tensor (3.20), the expression for a Laplacian: $\mathrm{d} \tilde{\star}_{9-p} \mathrm{~d} \alpha=(-1)^{p} \tilde{\nabla}^{2} \alpha \tilde{\star}_{9-p} 1$ and the relation $\star_{10}\left(A_{n} \wedge B_{m}\right)=(-1)^{n(9-p-m)} \star_{p+1} A_{n} \wedge \star_{9-p} B_{m}$, where $A_{n}$ is an external $n$-form and $B_{m}$ an internal $m$-form.
} 
We used in the second equation that a regular harmonic function on a compact space is constant and in the last equation we have pulled out the warp factor in $|\tilde{H}|^{2}$ and $\tilde{\delta}(O p) .{ }^{5}$

Summary of the solution. The Minkowski solution obtained in the smeared limit allows a localisation by adding a warp factor, $A$, in the metric Ansatz (3.18), (3.21). The varying dilaton can be written in terms of the warp factor via $(3.26)$, and the $F_{8-p}$ field strength can also be written in terms of the warp factor via (3.22), (3.24). The value of the warpfactor itself is then determined by the orientifold charge (and related fluxes) through equation (3.27).

As announced earlier, when $p=2$, there is a subtlety since $F_{4}$ and $F_{6}$ are each others dual and the solution needs some adjustment

$$
F_{4}=\mathrm{e}^{-\frac{3}{4} \phi} \star_{7} H+\mathrm{e}^{-6 A+\frac{1}{2} \phi} \star_{10} \tilde{\star}_{7} \mathrm{~d} \alpha .
$$

If one uses the expression for the dilaton in terms of the warp factor (3.26), then one finds that the BPS equation (3.13) has not changed since

$$
F_{6-p}=(-1)^{p} \mathrm{e}^{-\frac{p+1}{4} \phi} \star_{9-p} H=(-1)^{p} \mathrm{e}^{-\frac{p+1}{4} \phi_{0} \tilde{\star}_{9-p} H,}
$$

where the first Hodge star is taken with respect to the warped metric. Therefore the geometric moduli that are fixed by this BPS equation (3.6) have not shifted position due to the warping. The interpretation of this in terms of an effective potential $V_{\text {eff }}$ is that, at the Minkowski point, the contribution in $V_{\text {eff }}$ coming from the warped metric cancels the source contribution of $F_{8-p}$ in $V_{\text {eff }}$ (see also [7]). The integrated version of equation (3.27) also implies that (3.15) is still valid after localisation. Furthermore, the condition (3.14) remains unchanged, while the internal space changes from Ricci-flat (3.16) to conformally Ricci-flat (3.25).

Finally, we mention that for $p=6$ this solution is related to the "massive D6 solution" of [27], which considered the same setup, but in a non-compact setting where the O6 is replaced by a D6. This probably implies that a non-compact version, for which the $\mathrm{O} p$ source is replaced by a $\mathrm{D} p$ source exists for all values of $p$ we considered here, which generalises some results in [27].

\section{BPS solutions with negatively curved twisted tori}

Consider the setup above with a smeared $\mathrm{O} p$-plane whose tadpole is canceled by $H$ - and $F_{6-p}$-flux. A formal T-duality along one direction of the $F_{6-p}$-flux or one direction along the $\mathrm{O} p$-plane maps this setup into a smeared $\mathrm{O}(p \pm 1)$-plane whose tadpole is canceled by $H$ - and $F_{6 \mp 1-p}$-flux. Now we want to study cases that arise after one T-duality along the direction of the $H$-flux. If we start out with a torus as compact transverse space, then this leads to twisted tori that have negative curvature. Therefore, these setups are directly addressing the issue of [24]. There the authors show that compactifications on spaces with

\footnotetext{
${ }^{5}$ The delta function is proportional to $1 / \sqrt{g^{(9-p)}}$. This means that we have set the overall volume of the transverse space to one when we take the smeared limit $\delta(O p) \rightarrow 1$.
} 
negative curvature that lead to $\mathrm{dS}$ or Minkowski solutions require a warp factor whose contribution to the equations of motion is comparable to the fluxes everywhere in the compact space.

We assume that the entire $H$-flux has one leg along the last coordinate which we call the 9-direction i.e. $H=H_{i j 9} d x^{i} \wedge d x^{j} \wedge d x^{9}$. Furthermore, we assume that our space has a $\mathrm{U}(1)$ isometry (at least in the smeared case) corresponding to shifts of $x^{9}$. Then we can perform a T-duality [28, 29] along this direction and find a new space that can be conveniently written [30] in terms of the 1-forms $d x^{\mu}, d x^{i}, e^{9}=d x^{9}+\frac{1}{2} f_{i j}^{9} x^{i} d x^{j}$ with $\mu=0, \ldots, p-1, i=p, \ldots, 8$ and $f_{i j}^{9}=H_{i j 9}$. The T-dual setup has vanishing $H$-flux but non-vanishing $f_{i j}^{9}$ which is often referred to as metric flux. Note that $e^{9}$ is not closed but we rather have $\mathrm{d} e^{9}=\frac{1}{2} f_{i j}^{9} d x^{i} \wedge d x^{j}$. Motivated by this T-duality, we consider an $\mathrm{O} p$-plane along $\mu=0,1, \ldots, p-1$ and the $e^{9}$ direction and make the following Ansatz for the warped metric

$$
\begin{aligned}
\mathrm{d} s^{2} & =\mathrm{d} s_{p}^{2}+g_{99} e^{9} e^{9}+\mathrm{d} s_{9-p}^{2}=g_{\mu \nu} \mathrm{d} x^{\mu} \mathrm{d} x^{\nu}+g_{99} e^{9} e^{9}+g_{i j} \mathrm{~d} x^{i} \mathrm{~d} x^{j} \\
& =\mathrm{e}^{2 A}\left(\tilde{g}_{\mu \nu} \mathrm{d} x^{\mu} \mathrm{d} x^{\nu}+\tilde{g}_{99} e^{9} e^{9}\right)+\mathrm{e}^{\frac{2(p+1)}{p-7}} A \tilde{g}_{i j} \mathrm{~d} x^{i} \mathrm{~d} x^{j}
\end{aligned}
$$

which is not block diagonal in the $d x$-basis since the $e^{9} e^{9}$ term gives contributions that mix the $\mathrm{d} x^{9}$ and $\mathrm{d} x^{i}$ directions. ${ }^{6}$ Therefore, the Ricci tensor given in (3.20) is modified. It now has three contributions. One from the unwarped metric $\tilde{g}_{a b}$, another from the metric fluxes $f_{i j}^{9}$ and a third from the warp factor $A$. A lengthy calculation leads to

$$
\begin{aligned}
R_{\mu \nu} & =\tilde{R}_{\mu \nu}-\mathrm{e}^{\frac{16}{7-p} A} \tilde{g}_{\mu \nu} \tilde{\nabla}^{2} A, \\
R_{99} & =\frac{1}{2} \mathrm{e}^{\frac{32}{7-p} A} \tilde{g}_{99} \tilde{g}_{99}\left|\tilde{\mathrm{de}}^{9}\right|^{2}-\mathrm{e}^{\frac{16}{7-p} A} \tilde{g}_{99} \tilde{\nabla}^{2} A, \\
R_{9 i} & =\tilde{R}_{9 i}+\frac{8}{7-p} \mathrm{e}^{\frac{16}{7-p} A} \tilde{g}_{99}\left|e^{9} \wedge \mathrm{d} A \cdot \mathrm{d} e^{9}\right|_{9 i}^{\text {unwarped }} \\
R_{i j} & =\tilde{R}_{i j}-\frac{1}{2} \mathrm{e}^{\frac{16}{7-p} A} \tilde{g}_{99}\left|\tilde{\mathrm{d}}^{9}\right|_{i j}^{2}-\frac{p+1}{p-7} \tilde{g}_{i j} \tilde{\nabla}^{2} A+\frac{8(p+1)}{p-7} \partial_{i} A \partial_{j} A,
\end{aligned}
$$

where $\left|A_{n} \cdot B_{n}\right|_{a b}^{\text {unwarped }}=\frac{1}{(n-1) !} A_{a c_{1} \ldots c_{n-1}} B_{b d_{1} \ldots d_{n-1}} \tilde{g}^{c_{1} d_{1}} \ldots \tilde{g}^{c_{n-1} d_{n-1}}$, and we assume that the metric (4.1) has $\tilde{R}_{99}=0$. With this information we can now solve the equations of motions.

\subsection{The smeared solutions}

In this section we solve the equations of motions for a smeared $\mathrm{O} p$-plane i.e. in the nonwarped case $A=0$ with $\delta(O p) \rightarrow 1$. The non-zero fields are

$$
\phi_{0}=\text { cst. }, \quad F_{8-p}=m_{7-p} \wedge e^{9}, \quad R_{\mu \nu}, \quad R_{99}, \quad R_{9 i}, \quad R_{i j},
$$

\footnotetext{
${ }^{6}$ Note that, unlike in a usual vielbein basis, $e^{9}$ does not have unit norm as $g_{99}$ is in general not equal to one. Furthermore, all tensors with an index 9 are always meant to be with respect to the basis form $e^{9}$ rather than $\mathrm{d} x^{9}$.
} 
where $m_{7-p}$ is a closed $(7-p)$-form. The rest of the RR-fields and the $H$-flux are zero. Again $p=1, \ldots, 6$ and some equations require minor modifications for $p=3$ due to the self-duality of $F_{5}=(1+\star) m_{4} \wedge e^{9}$.

We start with the dilaton equation of motion for the smeared case,

$$
0=\nabla^{2} \phi_{0}=\frac{p-3}{4} \mathrm{e}^{\frac{p-3}{2} \phi_{0}}\left|F_{8-p}\right|^{2}-\frac{p-3}{4} \mathrm{e}^{\frac{p-3}{4} \phi_{0}} \mu_{p} .
$$

Using this in the external Einstein equation,

$$
R_{\mu \nu}=-\frac{7-p}{16} \mathrm{e}^{\frac{p-3}{2} \phi_{0}} g_{\mu \nu}\left|F_{8-p}\right|^{2}+\frac{7-p}{16} \mathrm{e}^{\frac{p-3}{4} \phi_{0}} g_{\mu \nu} \mu_{p}
$$

one finds $R_{\mu \nu}=0 .^{7}$ This means that our setup only allows for $p$-dimensional Minkowski solutions.

The Bianchi identity for $F_{8-p}$ is

$$
\mathrm{d} F_{8-p}=-(-1)^{p} m_{7-p} \wedge \mathrm{d} e^{9}=-\mu_{p} \epsilon_{9-p} .
$$

Together with (4.4) this gives

$$
-\star_{9-p} \mathrm{~d} F_{8-p}=\mathrm{e}^{\frac{p-3}{4} \phi_{0}}\left|F_{8-p}\right|^{2} .
$$

The Einstein equation in the 9-direction is

$$
\begin{aligned}
R_{99} & =\frac{1}{2} g_{99} g_{99}\left|\mathrm{~d} e^{9}\right|^{2} \\
& =\mathrm{e}^{\frac{p-3}{2} \phi_{0}}\left(\frac{1}{2}\left|F_{8-p}\right|_{99}^{2}-\frac{7-p}{16} g_{99}\left|F_{8-p}\right|^{2}\right)+\frac{7-p}{16} \mathrm{e}^{\frac{p-3}{4} \phi_{0}} g_{99} \mu_{p} \\
& =\mathrm{e}^{\frac{p-3}{2} \phi_{0}} \frac{1}{2}\left|F_{8-p}\right|_{99}^{2},
\end{aligned}
$$

where we used (4.4). Since for $F_{8-p}=m_{7-p} \wedge e^{9}$ one has $\left|F_{8-p}\right|_{99}^{2}=\left|F_{8-p}\right|^{2} g_{99}$ we find

$$
g_{99}\left|\mathrm{~d} e^{9}\right|^{2}=\mathrm{e}^{\frac{p-3}{2} \phi_{0}}\left|F_{8-p}\right|^{2} .
$$

Together with (4.7) this leads to

$$
g_{99} \star_{9-p} \mathrm{~d} e^{9} \wedge \mathrm{d} e^{9}=\mathrm{e}^{\frac{p-3}{2} \phi_{0}} g^{99} \star_{9-p} m_{7-p} \wedge m_{7-p}=\mathrm{e}^{\frac{p-3}{4} \phi_{0}}(-1)^{p} m_{7-p} \wedge \mathrm{d} e^{9},
$$

which implies

$$
\mathrm{d} e^{9}=(-1)^{p} g^{99} \mathrm{e}^{\frac{p-3}{4} \phi_{0}} \star_{9-p} m_{7-p} .
$$

This is of course nothing but the T-dual version of the BPS condition (3.6).

The Einstein equations for the directions transverse to the $\mathrm{O} p$-plane are

$$
\begin{aligned}
R_{i j} & =\tilde{R}_{i j}-\frac{1}{2} g_{99}\left|\mathrm{~d} e^{9}\right|_{i j}^{2} \\
& =\mathrm{e}^{\frac{p-3}{2} \phi_{0}}\left(\frac{1}{2}\left|F_{8-p}\right|_{i j}^{2}-\frac{7-p}{16} g_{i j}\left|F_{8-p}\right|^{2}\right)-\frac{p+1}{16} \mathrm{e}^{\frac{p-3}{4} \phi_{0}} g_{i j} \mu_{p} \\
& =\mathrm{e}^{\frac{p-3}{2} \phi_{0}}\left(\frac{1}{2}\left|F_{8-p}\right|_{i j}^{2}-\frac{1}{2} g_{i j}\left|F_{8-p}\right|^{2}\right) .
\end{aligned}
$$

\footnotetext{
${ }^{7}$ For the special case of $p=3$ we assume a Ricci-flat external space, because this is no longer implied by (4.4) and (4.5).
} 
Using (4.11) we can rewrite this as

$$
\tilde{R}_{i j}=\frac{1}{2} \mathrm{e}^{\frac{p-3}{2} \phi_{0}} g^{99}\left(\left|\star_{9-p} m_{7-p}\right|_{i j}^{2}+\left|m_{7-p}\right|_{i j}^{2}-g_{i j}\left|m_{7-p}\right|^{2}\right)=0 .
$$

Note that $\tilde{R}_{i j}=0$ does not mean that we have no curvature since $R_{i j} \neq 0$ and $R_{99} \neq 0$. The final Einstein equation reads $\tilde{R}_{9 i}=0$.

All other equations of motion are trivially satisfied, so that we have spelled out all the non-trivial equations of motion.

Summary of the solution. The a priori non-zero fields in the Ansatz for a smeared $\mathrm{O} p$-plane with $p=1, \ldots, 6$ and metric (4.1) (with $A=0$ ) are

$$
\phi_{0}=\text { cst. }, \quad F_{8-p}=m_{7-p} \wedge e^{9}, \quad R_{\mu \nu}, \quad R_{99}, \quad R_{9 i}, \quad R_{i j} .
$$

The equations of motion only allow for $p$-dimensional Minkowski solutions $\left(R_{\mu \nu}=0\right)$ modulo the caveat for $p=3$ mentioned in footnote 7 . All Bianchi identities and equations of motion can be reduced to $\tilde{R}_{9 i}=0$ and the following two conditions:

- A duality condition between the curvature, which is encoded in the non-closure of the 1 -form $e^{9}$, and the RR-flux

$$
\mathrm{d} e^{9}=(-1)^{p} g^{99} \mathrm{e}^{\frac{p-3}{4} \phi_{0}} \star_{9-p} m_{7-p} .
$$

- The amount of flux, and, as implied by (4.15), also the curvature are fixed by the Op-plane charge,

$$
\mu_{p}=\mathrm{e}^{\frac{p-3}{4} \phi_{0}}\left|F_{8-p}\right|^{2}=\mathrm{e}^{-\frac{p-3}{4} \phi_{0}} g_{99}\left|\mathrm{~d} e^{9}\right|^{2} .
$$

After giving a simple example we will proceed to show that these solutions can be localised by introducing a warp factor and allowing the dilaton to vary.

\subsection{A simple example}

There are many examples of so called twisted tori and coset spaces that can be used to obtain explicit Minkowski solutions of the type described above. Probably the simplest case is the one where the metric fluxes satisfy the Heisenberg algebra. Such a space can be easily compactified and is then T-dual to a three-torus with $H$-flux [31]. To obtain a simple solution we choose the unwarped metric to be $\tilde{g}_{\mu \nu}=\eta_{\mu \nu}, \tilde{g}_{99}=1$, and $\tilde{g}_{i j}=\delta_{i j}$. An explicit example that solves the equations of motion is then an $\mathrm{O} p$-plane with $p=1, \ldots, 6$ that wraps the directions $0,1, \ldots, p-1,9$, and the directions $p, p+1, \ldots, 8$ and 9 are compact. Furthermore, we choose the non-zero fields for our solution to be

$$
\begin{aligned}
& \phi_{0}=\text { cst., } F_{8-p}=(-1)^{p} \mathrm{e}^{-\frac{p-3}{4} \phi_{0}} f \mathrm{~d} x^{p} \wedge \mathrm{d} x^{p+1} \wedge \ldots \wedge \mathrm{d} x^{6} \wedge e^{9}, \\
& f_{78}^{9}=-f_{87}^{9}=f \Rightarrow R_{77}=R_{88}=-R_{99}=-\frac{1}{2} f^{2}, \quad \text { with } \quad f^{2}=\mathrm{e}^{\frac{p-3}{4} \phi_{0}} \mu_{p} .
\end{aligned}
$$

This is a $p$-dimensional Minkowski solution in which a smeared $\mathrm{O} p$-plane is compactified on an internal everywhere negatively curved space. As we explain in the following subsection, the $\mathrm{O} p$-plane can be localised if we introduce a warp factor and allow the dilaton to vary over the internal space. 


\subsection{The localised solutions}

To find localised solutions with $p=1, \ldots, 6$ we now include a non-zero warp-factor $A$ which we allow together with the dilaton $\phi$ to depend on all the coordinates transverse to the $\mathrm{O} p$-plane. For $F_{8-p}$ we make the Ansatz

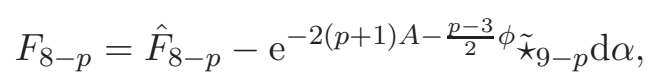

where $\hat{F}_{8-p}=m_{7-p} \wedge e^{9}$ is the $(8-p)$-form from (4.3) and $\alpha$ is a function of the transverse coordinates that will be determined below. The tilde will always mean the unwarped metric $\tilde{g}_{a b}$, the corresponding unwarped Hodge star $\tilde{\star}$, or contractions of forms done with $\tilde{g}_{a b}$. We start out by deriving a BPS condition similar to (3.23). The dilaton equation of motion

$$
\begin{aligned}
\nabla^{2} \phi & =\mathrm{e}^{\frac{2(p+1)}{7-p} A} \tilde{\nabla}^{2} \phi \\
& =\frac{p-3}{4} \mathrm{e}^{\frac{p-3}{2} \phi}\left(\left|\hat{F}_{8-p}\right|^{2}+\mathrm{e}^{\frac{2(p+1)(p-6)}{7-p} A-(p-3) \phi}(\tilde{\partial} \alpha)^{2}\right)-\frac{p-3}{4} \mathrm{e}^{\frac{p-3}{4} \phi} \mu_{p} \delta(O p),
\end{aligned}
$$

gives us an expression for $\tilde{\nabla}^{2} \phi$, and we find $\tilde{\nabla}^{2} A$ from the trace of the Einstein equations along the $\mathrm{O} p$-plane,

$$
\begin{aligned}
\tilde{g}^{\mu \nu} R_{\mu \nu}+\tilde{g}^{99} R_{99}= & \tilde{R}_{p}-\mathrm{e}^{\frac{16}{7-p} A}(p+1) \tilde{\nabla}^{2} A+\frac{1}{2} \mathrm{e}^{\frac{32}{7-p} A} \tilde{g}_{99}\left|\tilde{\mathrm{de}}^{9}\right|^{2} \\
= & -\frac{(7-p)(p+1)}{16} \mathrm{e}^{2 A+\frac{p-3}{2} \phi}\left(\left|\hat{F}_{8-p}\right|^{2}+\mathrm{e}^{\frac{2(p+1)(p-6)}{7-p} A-(p-3) \phi}(\tilde{\partial} \alpha)^{2}\right) \\
& +\frac{1}{2} \mathrm{e}^{\frac{p-3}{2} \phi}\left|\hat{F}_{8-p}\right|_{99}^{2}+\frac{(7-p)(p+1)}{16} \mathrm{e}^{2 A+\frac{p-3}{4} \phi} \mu_{p} \delta(O p) .
\end{aligned}
$$

Finally, from the Bianchi identity for $F_{8-p}$,

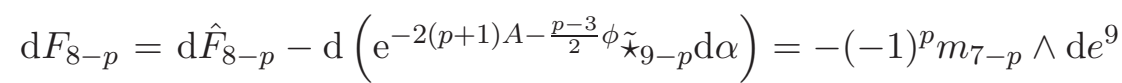

$$
\begin{aligned}
& -(-1)^{p}\left(\mathrm{e}^{\frac{(p-5)(p+1)}{7-p} A-\frac{p-3}{2} \phi} \tilde{\nabla}^{2} \alpha+\mathrm{e}^{\frac{(9-p)(p+1)}{7-p} A} \tilde{g}^{i j} \partial_{i}\left(\mathrm{e}^{-2(p+1) A-\frac{p-3}{2} \phi}\right) \partial_{j} \alpha\right) \epsilon_{9-p} \\
& =-\mu_{p} \delta(O p) \epsilon_{9-p},
\end{aligned}
$$

we get $\tilde{\nabla}^{2} \alpha$. Putting all these expressions together, we have

$$
\begin{aligned}
\tilde{\nabla}^{2}\left(\mathrm{e}^{(p+1) A+\frac{p-3}{4} \phi}\right. & \left.+(-1)^{p} \alpha\right)=\mathrm{e}^{\frac{(p-3)^{2}}{p-7} A+\frac{p-3}{4} \phi} \tilde{R}_{p} \\
+ & \mathrm{e}^{\frac{(p+1)(9-p)}{p-7} A-\frac{p-3}{4} \phi}\left|\partial\left(\mathrm{e}^{(p+1) A+\frac{p-3}{4} \phi}+(-1)^{p} \alpha\right)\right|^{2} \\
+ & \frac{1}{2} \mathrm{e}^{\frac{(p+1)(p-5)}{p-7} A+\frac{3(p-3)}{4} \phi}\left|\sqrt{g^{99}} m_{7-p}-(-1)^{p} \mathrm{e}^{-\frac{p-3}{4} \phi} \sqrt{g_{99} \star_{9-p}} \mathrm{~d} e^{9}\right|^{2},
\end{aligned}
$$

where the squares in the last two term are with respect to the warped metric. Integrating both sides over the compact space we find for Minkowski solutions that

$$
\alpha=(-1)^{p+1} \mathrm{e}^{(p+1) A+\frac{p-3}{4} \phi}+c s t .
$$

and

$$
\mathrm{d} e^{9}=(-1)^{p} \mathrm{e}^{\frac{p-3}{4} \phi} g^{99} \star_{9-p} m_{7-p} .
$$


Plugging the dilaton equation into the external Einstein equation we furthermore find

$$
0=\frac{4(p-3)}{7-p} \tilde{R}_{\mu \nu}=\tilde{g}_{\mu \nu} \mathrm{e}^{\frac{16}{7-p} A} \tilde{\nabla}^{2}\left(\frac{4(p-3)}{7-p} A-\phi\right) .
$$

Since a harmonic function on a compact space is constant, we have

$$
\phi=\frac{4(p-3)}{7-p} A+\phi_{0}
$$

which implies that the duality condition (4.11) is unchanged since

$$
\mathrm{d} e^{9}=(-1)^{p} \mathrm{e}^{\frac{p-3}{4} \phi} g^{99} \star_{9-p} m_{7-p}=(-1)^{p} \mathrm{e}^{\frac{p-3}{4} \phi_{0}} \tilde{g}^{99} \tilde{\star}_{9-p} m_{7-p} .
$$

Next we check the internal Einstein equations

$$
\begin{aligned}
R_{i j}= & \tilde{R}_{i j}-\frac{1}{2} \mathrm{e}^{\frac{16}{7-p} A} \tilde{g}_{99}\left|\tilde{\mathrm{d}}^{9}\right|_{i j}^{2}-\frac{p+1}{p-7} \tilde{g}_{i j} \tilde{\nabla}^{2} A+\frac{8(p+1)}{p-7} \partial_{i} A \partial_{j} A \\
= & \frac{1}{2} \partial_{i} \phi \partial_{j} \phi+\mathrm{e}^{\frac{p-3}{2} \phi}\left[\frac{1}{2}\left|\hat{F}_{8-p}\right|_{i j}^{2}+\frac{1}{2} \mathrm{e}^{-2(p+1) A-(p-3) \phi}\left(\tilde{g}_{i j}(\tilde{\partial} \alpha)^{2}-\partial_{i} \alpha \partial_{j} \alpha\right)\right. \\
& \left.-\frac{7-p}{16} \mathrm{e}^{\frac{2(p+1)}{p-7} A} \tilde{g}_{i j}\left(\left|\hat{F}_{8-p}\right|^{2}+\mathrm{e}^{\frac{2(p+1)(p-6)}{7-p} A-(p-3) \phi}(\tilde{\partial} \alpha)^{2}\right)\right] \\
& -\frac{(p+1)}{16} \mathrm{e}^{\frac{2(p+1)}{p-7} A+\frac{p-3}{4} \phi} \tilde{g}_{i j} \mu_{p} \delta(O p),
\end{aligned}
$$

which using (4.16), (4.20) and (4.26) reduces to (4.12).

There is one more non-trivial Einstein equation:

$$
\begin{aligned}
R_{9 i} & =\frac{8}{7-p} \mathrm{e}^{\frac{16}{7-p} A} \tilde{g}_{99}\left|e^{9} \wedge \mathrm{d} A \cdot \mathrm{d} e^{9}\right|_{9 i}^{\text {unwarped }} \\
& =\frac{8}{7-p} \mathrm{e}^{\frac{16}{7-p} A+\frac{p-3}{4} \phi_{0}}(-1)^{p}\left|e^{9} \wedge \mathrm{d} A \cdot \tilde{\star}_{9-p} m_{7-p}\right|_{9 i}^{\text {unwarped }},
\end{aligned}
$$

which is satisfied due to (4.27).

Finally, we have to make sure that the equation of motion for $F_{8-p}$ is satisfied i.e.

$$
\begin{aligned}
& 0=\mathrm{d}\left(\mathrm{e}^{\frac{p-3}{2} \phi} \star_{10} F_{8-p}\right)=\mathrm{d}\left(\mathrm{e}^{\frac{p-3}{2} \phi} \star_{10} \hat{F}_{8-p}\right)-\mathrm{d}\left((-1)^{p^{\star_{p+1}}} 1 \wedge \mathrm{d} \alpha\right) \\
& =\frac{16}{7-p} \mathrm{e}^{\frac{16}{7-p} A+\frac{p-3}{2} \phi_{0}} \mathrm{~d} A \wedge \epsilon_{p} \wedge\left(-(-1)^{p} \sqrt{\tilde{g}^{99}} \tilde{\star}_{9-p} m_{7-p}+\sqrt{\tilde{g}_{99}} \mathrm{e}^{-\frac{p-3}{4} \phi_{0}} \mathrm{~d} e^{9}\right),
\end{aligned}
$$

where we have used (4.23) and (4.26). This equation is again satisfied due to (4.27).

All other equations of motion are trivially satisfied, so that we have spelled out all the non-trivial equations of motions.

As an important observation, we note, using (4.1) and (4.2), that

$$
\int \sqrt{g^{(10)}} R_{(10-p)}=\int \sqrt{\tilde{g}}\left\{-8 \frac{p+1}{7-p}(\tilde{\nabla} A)^{2}-\frac{1}{4} e^{\frac{16}{7-p} A} \tilde{g}_{99} \tilde{g}^{i j} \tilde{g}^{k l} f_{i k}^{9} f_{j l}^{9}\right\}<0,
$$

where the integral is over the $(10-p)$-dimensional internal space, and we have dropped total derivative terms. This is the integral of the full internal curvature computed from the 
warped metric components $g_{99}$ and $g_{i j}$ weighted with the full ten-dimensional metric determinant. This quantity is, up to a Weyl-rescaling, just minus the contribution of the internal curvature to the $p$-dimensional scalar potential. ${ }^{8}$ The last term describes the original negative curvature term of the twisted torus, now dressed with a warp factor. The first term is due to the non-constancy of the warp factor in the localised case and clearly vanishes in the smeared limit. Interestingly, both terms are manifestly negative (assuming $p<7$, as we do in this paper), even though the negative tension objects are now properly localised. This somewhat circumvents difficulties found in [24], where negative curvature spaces for uplifting potentials were argued to be problematic as the negative energy-momentum sources supporting them are really localized objects. It should be emphasized, however, that there is no real uplifting in the present context, as we are looking at Minkowski BPS solutions.

Summary of the solution. We have shown that the smeared solution of subsection 4.1 persists after localisation, if we introduce a warp factor $A$ and add a new term to the RR field strength $F_{8-p}$,

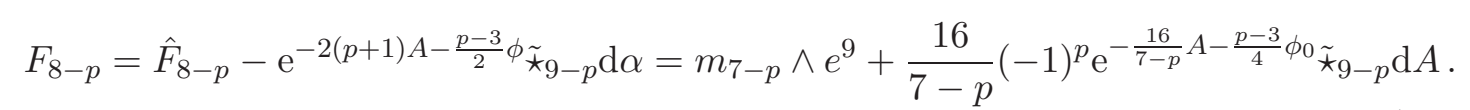

The only modulus that has changed is the dilaton that is no longer constant but rather proportional to the warp-factor

$$
\phi=\frac{4(p-3)}{7-p} A+\phi_{0} .
$$

The warp factor is determined for example through (4.19) which becomes for our choice of $\alpha(4.23)$

$$
\tilde{\nabla}^{2} \mathrm{e}^{\frac{16}{p-7} A}=-\mathrm{e}^{\frac{p-3}{2} \phi_{0}}\left|\tilde{\hat{F}}_{8-p}\right|^{2}+\mathrm{e}^{\frac{p-3}{4} \phi_{0}} \mu_{p} \tilde{\delta}(O p)
$$

where we have pulled out the warp factor dependence in $\left|\tilde{\hat{F}}_{8-p}\right|^{2}$ and $\tilde{\delta}(O p)$.

In [24] the authors have shown that compactifications down to dS or Minkowski space on everywhere negatively curved spaces must have significant warping and/or large stringy corrections. Since we have neglected stringy corrections but found compactifications that lead to Minkowski solutions, we can conclude that we have large warping everywhere. This means that the contributions of the warp factor are of the same order as the contributions of the fluxes and localised sources. This is apparent from (4.34). Far away from a localised source one could have naively expected that the warp factor approaches a constant and the warping becomes negligible. This would have made it impossible to find compactifications on negative curvature spaces that have a large volume i.e. in a regime where we can trust supergravity. However, as we can see from (4.34) the warp factor is everywhere sourced by the fluxes so that its contributions will be relevant everywhere even if the compact space is large.

As we saw in our particular example, however, the warping and the anisotropic conformal rescalings of the internal metric can conspire to still give a curvature contribution

\footnotetext{
${ }^{8}$ The inclusion of the $p$-dimensional part of the metric determinant is, in fact, important for the manifestly negative sign, as it cancels some remaining warp factors in front of the total derivative terms.
} 
to the $p$-dimensional scalar potential that is manifestly positive, showing that the situation can be more complex than expected from [24].

\section{Non-BPS solutions}

In the previous two sections we saw that the localization of smeared sources that satisfy a BPS condition leads to modifications of the solution, which, although important individually, still leave many features of the smeared solution unchanged when they are added up. As we will now show, in a non-BPS situation localisation corrections may in general lead to much more drastic effects.

\subsection{The smeared solutions}

To begin with, we look for non-BPS solutions with smeared sources, assuming that the dilaton is constant and the metric has the form of a direct product,

$$
\phi=\phi_{0}, \quad \mathrm{~d} s_{10}^{2}=\mathrm{d} s_{p+1}^{2}+\mathrm{d} s_{9-p}^{2} .
$$

The non-zero form fields are taken to be $H$ and $F_{6-p}$ with $p=2, \ldots, 6$, with the rest of the RR-fields being identically zero. It is convenient to pull out the part of $F_{6-p}$ that is along $\star_{9-p} H$ :

$$
F_{6-p}=\bar{F}_{6-p}+(-1)^{p} \mathrm{e}^{-\frac{p+1}{4} \phi_{0}} \kappa \star_{9-p} H,
$$

where $\bar{F}_{6-p}$ is a closed and co-closed form, satisfying $\bar{F}_{6-p} \wedge H=0$.

The Bianchi identity for $F_{8-p}=0$ then becomes

$$
0=\mathrm{e}^{-\frac{p+1}{4} \phi_{0}} \kappa|H|^{2} \epsilon_{9-p}-\mu_{p} \epsilon_{9-p} .
$$

So we have $\mathrm{e}^{-\frac{p+1}{4} \phi_{0}} \kappa|H|^{2}=\mu_{p}$ and therefore $\kappa>0$ for an orientifold plane and $\kappa<0$ for a D-brane corresponding to $\mu_{p}<0$. Using the $F_{8-p}$ Bianchi identity in the dilaton equation of motion one finds

$$
\left|\bar{F}_{6-p}\right|^{2}=\left(-\kappa^{2}+\frac{p-3}{p-1} \kappa+\frac{2}{p-1}\right) \mathrm{e}^{-\frac{p+1}{2} \phi_{0}}|H|^{2} .
$$

Since $\left|\bar{F}_{6-p}\right|^{2}$ and $|H|^{2}$ are internal and therefore positive we find the range $-\frac{2}{p-1} \leq \kappa \leq 1$. The external Einstein equation simplifies to

$$
R_{\mu \nu}=-\frac{1-\kappa}{2(p-1)} \mathrm{e}^{-\phi_{0}}|H|^{2} g_{\mu \nu}
$$

So we see that we have AdS solutions for $\kappa<1$ (the case $\kappa=1$ corresponds to the BPS Minkowski solutions discussed in section 3). The internal Einstein equation gives

$$
R_{i j}=-\frac{(1-\kappa)(1+\kappa(p-1))}{2(p-1)} e^{-\phi_{0}}|H|^{2} g_{i j}+\frac{1}{2} \mathrm{e}^{\frac{p-1}{2} \phi_{0}}\left|\bar{F}_{6-p}\right|_{i j}^{2}+\frac{1}{2}\left(1-\kappa^{2}\right) \mathrm{e}^{-\phi_{0}}|H|_{i j}^{2},
$$

assuming $\left|\bar{F}_{6-p} \cdot H\right|_{i j}=0$. Although the internal components of the Ricci tensor have no fixed sign, one finds from taking the trace

$$
R_{9-p}=\frac{(1-\kappa) p}{p-1} e^{-\phi_{0}}|H|^{2}=-\frac{2 p}{p+1} R_{p+1} .
$$

Since the external space is AdS the internal space has to be positively curved. 


\subsection{A simple example}

Let us consider the simple situation $\kappa=-\frac{2}{p-1}$, for which (5.4) implies $\bar{F}_{6-p}=0$, and we furthermore have a net D-brane charge,

$$
\mu_{p}=-\frac{2}{p-1} \mathrm{e}^{-\frac{p+1}{4} \phi_{0}}|H|^{2} .
$$

The value of the external Ricci scalar becomes

$$
R_{p+1}=-\frac{(p+1)^{2}}{2(p-1)^{2}} \mathrm{e}^{-\phi_{0}}|H|^{2},
$$

and the internal Einstein equation reduces to

$$
R_{i j}=\frac{p+1}{2(p-1)^{2}} \mathrm{e}^{-\phi_{0}}|H|^{2} g_{i j}+\frac{(p+1)(p-3)}{2(p-1)^{2}} \mathrm{e}^{-\phi_{0}}|H|_{i j}^{2} .
$$

To find simple explicit solutions we take the internal space to be a product of two spheres (cf. [32] for $p=3$ ),

$$
M_{9-p}=S^{3} \times S^{6-p},
$$

with volume forms $\epsilon_{3}$ and $\epsilon_{6-p}$. Then the solution reads

$$
\begin{aligned}
H & =h \epsilon_{3}, \\
F_{6-p} & =-\frac{2}{p-1}(-1)^{p} \mathrm{e}^{-\frac{p+1}{4} \phi_{0}} \star_{9-p} H=-\frac{2}{p-1} \mathrm{e}^{-\frac{p+1}{4} \phi_{0}} h \epsilon_{6-p},
\end{aligned}
$$

and the internal Einstein equation fixes the curvature radii (and hence the volume) of the $S^{3}$ and the $S^{6-p}$,

$$
\begin{aligned}
R_{i j}^{S^{3}} & =\frac{(p+1)(p-2)}{2(p-1)^{2}} \mathrm{e}^{-\phi_{0}} h^{2} g_{i j}^{S^{3}}, \\
R_{i j}^{S^{6-p}} & =\frac{p+1}{2(p-1)^{2}} \mathrm{e}^{-\phi_{0}} h^{2} g_{i j}^{S^{6-p}} .
\end{aligned}
$$

\subsection{The failure to localise?}

In order to argue that non-BPS solutions based on smeared sources should in general not be localisable, we study an explicit example. We show this for $\kappa=-\frac{2}{p-1}$, since then $\bar{F}_{6-p}=0$ due to $(5.4) .^{9}$

We will first consider the case with $p=3$ since we can easily compare the O3-plane solution of GKP [1] with $\kappa=1$, i.e. ISD flux, to a solution with a D3-brane and $\kappa=-1$, i.e. IASD fluxes. To this end, we write for the $F_{3}$-flux

$$
F_{3}=\mp \mathrm{e}^{-\phi} \star_{6} H
$$

where from here on the upper sign corresponds to the BPS solution and the lower sign to the non-BPS solution. Equation (5.16) is our starting point as we take it as the definition of the solution. Whether a less standard Ansatz might lead to localised solutions that reduce,

\footnotetext{
${ }^{9}$ For an interesting discussion of local solutions that are close to but not at the BPS point see [33].
} 
in the smeared limit, to the smeared solutions constructed above is not clear. One should however keep in mind that (5.16) fixes some of the moduli at certain values, so changing this condition could change the entire solution in a more non-trivial way. We leave this for future investigation [34].

As in the smeared solution, the fluxes satisfy the Bianchi identities

$$
\mathrm{d} H=\mathrm{d} F_{3}=0 .
$$

The most general metric Ansatz for the localised solution is

$$
\mathrm{d} s_{10}^{2}=\mathrm{e}^{2 A} \mathrm{~d} \tilde{s}_{4}^{2}+\mathrm{d} s_{6}^{2},
$$

where the internal metric $\mathrm{d} s_{6}^{2}$ is left arbitrary. With (5.16), the integrated dilaton equation of motion implies that $F_{1}$ vanishes, as there are no net orientifold charges for $p \neq 3$ in our setup. The dilaton equation then also implies that $\phi=\phi_{0}$ is constant. The Ansatz for $F_{5}$ reads $^{10}$

$$
F_{5}=-\left(1+\star_{10}\right) \mathrm{e}^{-4 A} \star_{6} \mathrm{~d} \alpha .
$$

Let us go through the equations of motion. The dilaton equation is automatically satisfied due to the duality condition (5.16). The equations of motion for $H$ and $F_{3}$ are solved if

$$
\alpha= \pm \mathrm{e}^{4 A}+\text { cst. }
$$

The different sign for the $F_{5}$ field in both cases makes sense since the source is an O3-plane in the BPS case and a D3-brane in the non-BPS case. The $F_{5}$ equation then gives

$$
4 \nabla^{2} A=\mathrm{e}^{-\phi_{0}}|H|^{2} \mp \mu_{3} \delta(O 3 / D 3) .
$$

Note, that $\mu_{3}>0$ for the O3-plane and $\mu_{3}<0$ for the D3-brane. The external Einstein equation is

$$
\mathrm{e}^{-2 A} \tilde{R}_{4}-4 \nabla^{2} A=-\mathrm{e}^{-\phi_{0}}|H|^{2}+\mu_{3} \delta(O 3 / D 3) .
$$

Combining these two equations leads to

$$
\mathrm{e}^{-2 A} \tilde{R}_{4}=(1 \mp 1) \mu_{3} \delta(O 3 / D 3)
$$

Clearly, in the BPS case, we recover the Minkowski solution $\tilde{R}_{4}=0$ while in the non-BPS case there is no AdS solution anymore! One simply goes away from the source and then this equation is inconsistent with an AdS solution $\left(\tilde{R}_{4}=-2|\Lambda|\right)$. Even if one tries to make sense out of this by regularising the delta function, one needs that $\mathrm{e}^{-2 A} \sim \delta_{\text {regularised }}(D 3)$, which is not true. Only in the smeared limit, where

$$
\delta(D 3) \rightarrow 1, \quad A \rightarrow 0,
$$

equation (5.23) makes sense since we reproduce the previous result (5.8), (5.9). Due to our mild assumptions we believe that our simple smeared solution ceases to exist once

\footnotetext{
${ }^{10}$ One can verify that this Ansatz is required by the self-duality of $F_{5}$ and the equations of motion for $F_{3}$ and $H$, which also imply that there cannot be any warp factor appearing in (5.16).
} 


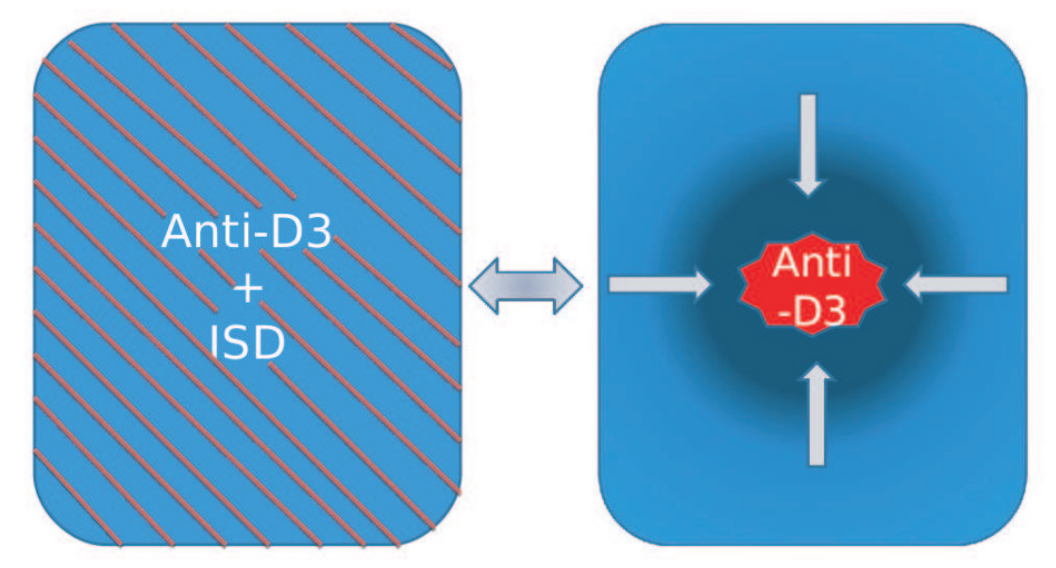

Figure 1. The smeared (left) versus localised (right) case.

localisation effects are taken into account. We should point out that a completely analogous localisation problem is encountered for the corresponding AdS solution with ISD fluxes and an anti-D3 brane.

For $p \neq 3$ one can likewise show that the smeared solutions with $\kappa=-\frac{2}{p-1}$, i.e. $\bar{F}_{6-p}=0$, have to get altered or even disappear upon taking the localisation effects into account: plugging the Ansatz $F_{6-p}=-\frac{2}{p-1}(-1)^{p} \mathrm{e}^{-\frac{p+1}{4} \phi} \star_{9-p} H$ into the $F_{6-p}$ and $H$ equations of motion for a generic $F_{8-p}$ and dilaton $\phi$ leads to a contradiction.

There is a simple physical picture behind these technical difficulties with localisation. As is well known I(A)SD fluxes act as smeared (anti-) D3 branes, from the point of view of their charge and energy-momentum. Therefore adding anti-D3 branes to ISD flux (or vice versa), creates a perturbative instability when we localise the anti-branes, as shown in figure 1. When the anti-branes are localised they single out a preferred point that attracts ISD fluxes. In the smeared case there is no preferred point of attraction, and the instability is only non-perturbative (brane-flux annihilation).

Even though our localised Ansatz is not the most general one (see discussion below (5.16)), this physical reasoning seems to strengthen our belief in that even the most general Ansatz would fail to give a solution.

\section{Discussion}

We have presented BPS solutions from compactifications on Ricci-flat and negatively curved spaces. The T-duality chain that relates these BPS solutions to each other is quite straightforward if one T-dualises the smeared GKP solution on a torus. ${ }^{11}$ To obtain the solutions with a Ricci-flat internal space one either takes the T-duality circle along the orientifold (going down in dimension) or on the torus along a cycle without $H$-flux. If we T-dualise a

\footnotetext{
${ }^{11}$ The Buscher rules $[28,29]$ need a U(1) symmetry, which requires us to smear the solutions.
} 
cycle with $H$-flux, we obtain a solution on a twisted torus. The solutions obtained in this way can again be T-dualised up or down giving rise to all the solutions we have presented. Characterising the solutions by their $D$-dimensional generalisation of the ISD condition, we have schematically

$$
H \propto \star_{9-p} F_{6-p} \underset{T_{F_{7-p}}}{\stackrel{T_{O p}}{\rightleftarrows}} H \propto \star_{10-p} F_{7-p} \underset{T_{e^{9}}}{\stackrel{T_{H}}{\rightleftarrows}} \mathrm{de} e^{9} \propto \star_{9-p} i_{9} F_{8-p}
$$

where the first pair of arrows indicates how we connect the various smeared solutions with Ricci-flat internal spaces and the second arrow pair connects the solutions with Ricci-flat internal spaces to the twisted tori. The latter solutions are characterised by a duality condition between the metric flux and the RR-flux.

By going through the equations of motion we demonstrated that the smeared BPS solutions can be localised ${ }^{12}$ and that the moduli do not shift (even for the smeared solutions with everywhere negative internal curvature). This is attributed to the BPS condition that makes the contributions from the localisation cancel against each other in the effective potential [7]. An intuitive argument for this is the "no-force" condition for mutually BPS objects. We have the ISD fluxes (and their T-dual generalisations) which act as a smeared source with positive charge and positive tension and the $\mathrm{O} p$-plane. General lore says that when such objects are combined in a mutually BPS way they will not affect each other due to the cancellation between gravitational forces and electromagnetic forces.

While we believe that solutions that do not get altered will be BPS, we do not claim that all BPS solutions with smeared orientifolds allow a localisation. It rather seems that the localisation does not always work in such a simple way for BPS solutions. It is for example not known whether or how the supersymmetric solutions of [35] are localisable, mainly because they involve multiple intersecting orientifolds. Localised solutions with similarly BPS intersecting $\mathrm{O} p$-planes or $\mathrm{D} p$-branes are not even known in ten-dimensional flat space.

We also studied simple non-BPS solutions where one generically expects problems when trying to localise smeared sources. Indeed, our most important result concerns the non-BPS AdS solutions, which we have derived in the smeared limit. ${ }^{13}$ To our knowledge this is the first time that such explicit solutions have been constructed. We have argued from a $10 \mathrm{D}$ point of view that the localisation procedure fails for these solutions so that they probably cease to exist.

If one studies flux compactifications from the point of view of an effective potential, one has to estimate the size of the individual localisation corrections (such as the warping correction). The separate terms (the one from the warp factor) were argued in [24] to be of the same order as the fluxes when one compactifies on everywhere negatively curved spaces. This was one of the motivations for the explicit presentation of the twisted tori solutions in this paper. We have generalised the existing localised orientifold solutions on twisted tori in two ways. First, we extended to Minkowski vacua of different dimensions.

\footnotetext{
${ }^{12}$ Note that this is more general than arguments that rely on integrability from supersymmetry. As in GKP [1] we also allow the solutions to break SUSY.

${ }^{13}$ In $D=4$ these are solutions with non-ISD fluxes.
} 
Second, our solutions are BPS-like but not necessarily SUSY, because we have analysed the full 10D equations of motion, instead of just the pure spinor equations. As an important application, we found that the properly integrated internal curvature stays negative, even after localisation of the orientifold planes (in fact, the warp factor gradients even introduce an additional negative term in the integrated internal curvature). We would like to point out that T-duality connects the twisted tori solutions to the GKP-like solutions. Therefore the arguments of [24] extends to Ricci-flat internal spaces with $H$-flux, even in the large volume limit. Regardless of the size of the warping, we have shown that the sum of the localisation corrections cancels in certain cases at the BPS points. However, we expect that smeared non-BPS solutions get changed or might even cease to exist when the localisation effects are taken into account. Concretely, what we have shown in an explicit example is that there is an incompatibility between having a static solution (stable or unstable) based on mutually non-BPS building blocks solving all the equations of motion, and sensible localisation. It would be interesting to understand in more detail how strong the back reaction of the localised source is, perhaps in the style of the investigations done in [36].

\section{Acknowledgments}

We like to thank Ralph Blumenhagen, Steve Giddings, Thomas Grimm, Michael Haack, Shamit Kachru, Paul Koerber, Luca Martucci, Liam McAllister, Gary Shiu and Jian Qiu for useful discussions and Bret Underwood for discussions and valuable comments on an earlier draft. U.D. is supported by the Swedish Research Council (VR) and the Göran Gustafsson Foundation. D.J., T.W. and M.Z. are supported by the German Research Foundation (DFG) within the Emmy Noether Program (Grant number ZA 279/1-2) and the Cluster of Excellence "QUEST". T.V.R. is supported by the Göran Gustafsson Foundation. T.W. is supported by the Alfred P. Sloan Foundation and by the NSF under grant PHY-0757868. This research was supported in part by the National Science Foundation under Grant No. NSF PHY05-51164.

\section{References}

[1] S.B. Giddings, S. Kachru and J. Polchinski, Hierarchies from fluxes in string compactifications, Phys. Rev. D 66 (2002) 106006 [hep-th/0105097] [SPIRES].

[2] M.B. Schulz, Superstring orientifolds with torsion: O5 orientifolds of torus fibrations and their massless spectra, Fortsch. Phys. 52 (2004) 963 [hep-th/0406001] [SPIRES].

[3] M. Graña, R. Minasian, M. Petrini and A. Tomasiello, A scan for new $N=1$ vacua on twisted tori, JHEP 05 (2007) 031 [hep-th/0609124] [SPIRES].

[4] C. Angelantonj, S. Ferrara and M. Trigiante, New $D=4$ gauged supergravities from $N=4$ orientifolds with fluxes, JHEP 10 (2003) 015 [hep-th/0306185] [SPIRES].

[5] J.-P. Derendinger, C. Kounnas, P.M. Petropoulos and F. Zwirner, Superpotentials in IIA compactifications with general fluxes, Nucl. Phys. B 715 (2005) 211 [hep-th/0411276] [SPIRES]. 
[6] L. Randall and R. Sundrum, A large mass hierarchy from a small extra dimension, Phys. Rev. Lett. 83 (1999) 3370 [hep-ph/9905221] [SPIRES].

[7] O. DeWolfe and S.B. Giddings, Scales and hierarchies in warped compactifications and brane worlds, Phys. Rev. D 67 (2003) 066008 [hep-th/0208123] [SPIRES].

[8] S.B. Giddings and A. Maharana, Dynamics of warped compactifications and the shape of the warped landscape, Phys. Rev. D 73 (2006) 126003 [hep-th/0507158] [SPIRES].

[9] L. Martucci, D-branes on general $N=1$ backgrounds: superpotentials and D-terms, JHEP 06 (2006) 033 [hep-th/0602129] [SPIRES].

[10] A.R. Frey and A. Maharana, Warped spectroscopy: localization of frozen bulk modes, JHEP 08 (2006) 021 [hep-th/0603233] [SPIRES].

[11] P. Koerber and L. Martucci, From ten to four and back again: how to generalize the geometry, JHEP 08 (2007) 059 [arXiv:0707.1038] [SPIRES].

[12] M.R. Douglas and G. Torroba, Kinetic terms in warped compactifications, JHEP 05 (2009) 013 [arXiv:0805.3700] [SPIRES].

[13] G. Shiu, G. Torroba, B. Underwood and M.R. Douglas, Dynamics of warped flux compactifications, JHEP 06 (2008) 024 [arXiv: 0803.3068] [SPIRES].

[14] A.R. Frey, G. Torroba, B. Underwood and M.R. Douglas, The universal Kähler modulus in warped compactifications, JHEP 01 (2009) 036 [arXiv:0810.5768] [SPIRES].

[15] F. Marchesano, P. McGuirk and G. Shiu, Open string wavefunctions in warped compactifications, JHEP 04 (2009) 095 [arXiv: 0812.2247] [SPIRES].

[16] L. Martucci, On moduli and effective theory of $N=1$ warped flux compactifications, JHEP 05 (2009) 027 [arXiv:0902.4031] [SPIRES].

[17] H.-Y. Chen, Y. Nakayama and G. Shiu, On D3-brane dynamics at strong warping, Int. J. Mod. Phys. A 25 (2010) 2493 [arXiv:0905.4463] [SPIRES].

[18] M.R. Douglas, Effective potential and warp factor dynamics, JHEP 03 (2010) 071 [arXiv: 0911.3378] [SPIRES].

[19] S. Kachru, M.B. Schulz, P.K. Tripathy and S.P. Trivedi, New supersymmetric string compactifications, JHEP 03 (2003) 061 [hep-th/0211182] [SPIRES].

[20] S. Gurrieri, J. Louis, A. Micu and D. Waldram, Mirror symmetry in generalized Calabi-Yau compactifications, Nucl. Phys. B 654 (2003) 61 [hep-th/0211102] [SPIRES].

[21] D. Lüst, F. Marchesano, L. Martucci and D. Tsimpis, Generalized non-supersymmetric flux vacua, JHEP 11 (2008) 021 [arXiv:0807.4540] [SPIRES].

[22] T. Banks, TASI lectures on holographic space-time, SUSY and gravitational effective field theory, arXiv:1007.4001 [SPIRES].

[23] S.M. Carroll, M.C. Johnson and L. Randall, Dynamical compactification from de Sitter space, JHEP 11 (2009) 094 [arXiv:0904.3115] [SPIRES].

[24] M.R. Douglas and R. Kallosh, Compactification on negatively curved manifolds, JHEP 06 (2010) 004 [arXiv: 1001.4008] [SPIRES].

[25] P. Koerber, Lectures on generalized complex geometry for physicists, arXiv:1006.1536 [SPIRES]. 
[26] U.H. Danielsson, S.S. Haque, G. Shiu and T. Van Riet, Towards classical de Sitter solutions in string theory, JHEP 09 (2009) 114 [arXiv:0907. 2041] [SPIRES].

[27] B. Janssen, P. Meessen and T. Ortín, The D8-brane tied up: String and brane solutions in massive type IIA supergravity, Phys. Lett. B 453 (1999) 229 [hep-th/9901078] [SPIRES].

[28] T.H. Buscher, A symmetry of the string background field equations, Phys. Lett. B 194 (1987) 59 [SPIRES].

[29] S.F. Hassan, $\mathrm{SO}(d, d)$ transformations of Ramond-Ramond fields and space- time spinors, Nucl. Phys. B 583 (2000) 431 [hep-th/9912236] [SPIRES].

[30] J. Shelton, W. Taylor and B. Wecht, Nongeometric flux compactifications, JHEP 10 (2005) 085 [hep-th/0508133] [SPIRES].

[31] M.R. Douglas and S. Kachru, Flux compactification, Rev. Mod. Phys. 79 (2007) 733 [hep-th/0610102] [SPIRES].

[32] E. Silverstein, TASI/PiTP/ISS lectures on moduli and microphysics, hep-th/0405068 [SPIRES].

[33] D. Baumann, A. Dymarsky, S. Kachru, I.R. Klebanov and L. McAllister, D3-brane potentials from fluxes in AdS/CFT, JHEP 06 (2010) 072 [arXiv: 1001.5028] [SPIRES].

[34] J. Blaback, U. Danielsson, D. Junghans, T. Van Riet, T. Wrase and M. Zagermann. work in progress.

[35] O. DeWolfe, A. Giryavets, S. Kachru and W. Taylor, Type IIA moduli stabilization, JHEP 07 (2005) 066 [hep-th/0505160] [SPIRES].

[36] I. Bena, M. Graña and N. Halmagyi, On the existence of meta-stable vacua in Klebanov-Strassler, JHEP 09 (2010) 087 [arXiv: 0912.3519] [SPIRES]. 